\title{
Subcutaneous basal cell carcinoma: A case report
}

\author{
Tenley Voris MD, Robert Quinn MD, Mohammed Elahi MD, Hashim Alani MD
}

\begin{abstract}
T Voris, R Quinn, M Elahi, H Alani. Subcutaneous basal cell carcinoma: A case report. Can J Plast Surg 2003;11(2):85-86.

An unusual case of a patient who presented with a subcutaneous soft tissue lesion of the back is described. The patient had no preceding history of cutaneous malignancy or local trauma. Excision of the lesion revealed a diagnosis consistent with basal cell carcinoma with deep infiltration. The site was re-excised with a generous margin to ensure complete removal. There has been no recurrence for 18 months. This case is presented to underscore the rarity and importance of its existence.
\end{abstract}

Key Words: Basal cell cancer; Subcutaneous; Subdermal

\section{Rapport de cas d'un carcinome basocellulaire sous-cutané}

Est décrit le cas inhabituel d'un patient qui s'est présenté avec une lésion des tissus mous sous-cutanés dans le dos. Le patient ne présentait aucun antécédent de malignité cutanée ou de traumatisme local. L'excision de la lésion a révélé un diagnostic confirmant la présence d'un carcinome basocellulaire avec infiltration profonde. Une nouvelle excision, comportant une marge plus importante, a été effectuée au foyer de la lésion afin d'en garantir l'extraction complète. Aucune récurrence ne s'est manifestée depuis 18 mois. Ce cas est présenté pour en souligner la rareté et l'importance.
$\mathrm{B}_{\mathrm{m}}^{\mathrm{a}}$ sal cell carcinoma (BCC) is the most common cutaneous malignancy that humans acquire in the course of a lifetime (1). Approximately $85 \%$ of all BCCs appear in the head and neck region, with only $10 \%$ occurring in the torso and trunk $(2,3)$. Basal cell lesions that occur in areas not exposed to the sun occasionally have a propensity for aggressive and locally invasive behavior. This characteristic underscores the importance of wide local excision to achieve excisional control and to avoid recurrence. The following report depicts an unusual case of a patient who presented with a subcutaneous soft tissue lesion of the back. Excision of the lesion revealed a diagnosis consistent with basal cell carcinoma with deep infiltration.

\section{CASE PRESENTATION}

A 21-year-old white woman presented in June 2000 with a soft tissue mass near the midline of the upper back. The lesion had been slowly enlarging over the preceding six months to the point that it was noticeable to the patient during activities of daily living. There was no pain or tenderness associated with the mass. On physical examination, a $1.2 \mathrm{~cm} \times 1.2 \mathrm{~cm}$ subcutaneous lesion was identified with no evidence of overlying skin abnormality. A presumptive diagnosis of lipoma was made followed by its direct excision. During the surgical resection, dissection was more arduous; the specimen exhibited a deeper color and did not have the discrete boundaries that are often seen with lipomas. Serial pathological sections revealed an intact epidermis with a deeply infiltrating BCC at the level of the dermis. The tumour reached both lateral lines of resection, including the deep subcutaneous margin. Mitotic figures were not attached to the epidermis. After confirmation of the diagnosis from a second pathologist, the patient was returned to the operating theatre for re-excision. A wide resection of the lesion was performed, measuring $5 \mathrm{~cm} \times 2.5 \mathrm{~cm}$ at the skin surface. The dissection was carried into the deep subcutaneous tissue, taking care to ensure an adequate cuff of uninvolved tissue for the margin. Frozen section control was achieved followed by primary closure. Permanent pathology revealed nests of BCC present in the deep dermis and superficial subcutaneous fat (Figure 1). The deep and lateral excisional margins were reported as being negative for tumour. Nodular BCC was the final diagnosis. There has been no tumour recurrence 18 months postoperatively.

\section{DISCUSSION}

BCC is the most commonly occurring skin cancer in humans, and its incidence appears to be increasing $(1,3)$. The vast majority of patients develop BCC on sun-exposed areas of the skin, in direct proportion to the number of pilosebaceous units present (4). Fair-skinned, blue-eyed people engaged in outdoor occupations or activities have a higher incidence of these tumours. Ultraviolet light exposure, radiation, arsenic exposure and genetic factors play an important role in the developmental etiology, but in some cases, no cause can be ascribed. Sun-exposed areas of the head and neck region account for upwards of $85 \%$ of all BCCs (4-6). The most commonly occurring sites are the nose $(26 \%)$, the cheek $(16 \%)$, the periorbital region $(14 \%)$, the scalp $(11 \%)$ and the ear and periauricular regions $(11 \%)(4-6)$.

Several classifications of BCC have been described in the literature and are in common use in clinical practice today. Four clinical types are generally recognized: superficial, nodular, pigmented and morphea-like or sclerosing. The latter variety is by far the most dangerous form. The margins are normally indistinct and the lesion may go undetected for years.

Division of Plastic and Reconstructive Surgery, Providence Hospital, Southfield, Michigan 48075, USA

Correspondence and reprints: Dr Robert Quinn, Third Floor, Fischer Center, Providence Hospital, 16001 West Nine Mile Road, Southfield, Michigan 48075, USA. Telephone 248-424-5883, fax 248-424-5881, e-mail tvoris@hotmail.com 


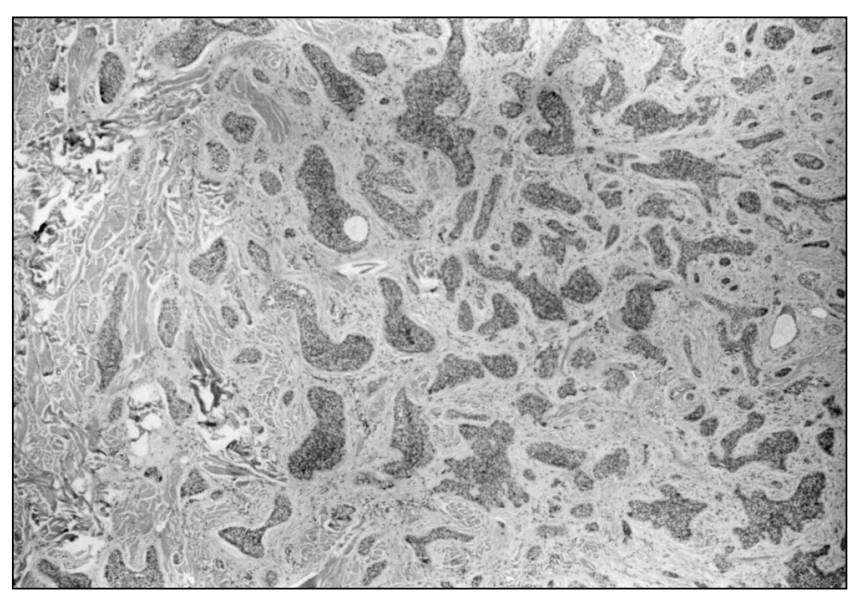

Figure 1) Permanent pathology slide shows nests of basal cell carcinoma present in the deep dermis and superficial subcutaneous fat

In contrast, the other clinical types normally have a discrete cutaneous component that is well demarcated, allowing for easier surveillance and management. Histological classifications more or less parallel the clinical subtypes and include solid, keratotic, cystic and adenoid patterns (7). In the solid pattern, masses of basal cells with minimal connective tissue stroma and no cell differentiation is seen, whereas in the keratotic variety, the BCCs differentiate toward hair structures. Cystic tumours show differentiation toward sebaceous glands with one or several cystic spaces within the tumour lobules. In the adenoid variety, the tumour reveals a glandular or tubular formation with epithelial strands that form a lace-like pattern. In some instances, BCC will show evidence of multiple histopathological forms combined $(7,8)$.

From a histological perspective, BCCs arise from the basal layer of the epidermis and from the pilosebaceous adnexa (4). Tumour proliferation results in three dimensional growth that ultimately manifests with an early cutaneous eruption that is described according to its clinical appearance. Volume-mass distortion of the surrounding tissues accompanies the skin presentation. The present case report suggests deep and peripheral growth in the absence of superficial extension. Although exceedingly rare, it certainly stands to reason that this pattern of growth is plausible and a reasonable explanation in this patient.

Most BCCs are easily treated small skin lesions that occur on sun-exposed areas of the skin. Occasionally, BCC will display aggressive histological features including infiltrative growth patterns, shown in the patient presented. These tumours fall into the subgroup category of aggressive growth BCC (9). This variant defines a group of basal cell cancers that are both clinically and histologically aggressive. These tumours normally occur in sun-protected regions of the body and are more likely in patients under 35 years of age. The aggressive growth BCCs are typically defined with the following criteria: epithelial islands separated by increased stroma; poorly defined peripheral palisading of tumour lobules; epithelial spikes; infiltrative rather than expansile behavior; and having the inherent potential for invasion (9).

The form of therapy chosen to treat these lesions plays an important role in the development of recurrent disease. Wide local excision with pathological confirmation of $5 \mathrm{~mm}$ to $10 \mathrm{~mm}$ margins constitutes a safe approach. Inadequate treatment will lead to recurrence and reconstructive difficulties that otherwise could have been avoided. The commonly accepted five-year recurrence rate varies widely between $5 \%$ to $40 \%$ and is a function of several tumour characteristics including location, histological subtype, tumour size and adequacy of resection (2-4). Although metastases are extremely rare, these lesions can invade by direct extension and may result in significant morbidity and even death by invasion into vital structures or from superimposed infections $(8,9)$.

\section{CONCLUSIONS}

The patient presented in the present case report fits into multiple categories reviewed in the literature. This is a unique case in that a subcutaneous BCC was diagnosed for the presentation of a soft tissue lesion of the back with an intact epidermal covering. The pathology demonstrated a deeply infiltrative and aggressive $\mathrm{BCC}$ without involvement of the epidermis. Re-excision was performed with negative tumour margins. There has been no tumour recurrence 18 months postoperatively. Due to the aggressive potential for these types of tumours, excision with histological confirmation of the margins is the recommended approach to treatment.

\section{REFERENCES}

1. Silverberg E, Lubera J. Cancer statistics, 1987. CA Cancer J Clin 1987;37:2-19.

2. Headington JT. Epidermal carcinomas of the integument of the nose and ear. In: Batsakis JG, editor. Tumors of the Head and Neck. Baltimore: Williams \& Wilkins, 1979:420.

3. Levine HL, Bailin PL. Basal cell carcinoma of the head and neck: Identification of the high risk patient. Laryngoscope 1980;90:955

4. Swanson NA. Basal cell carcinoma: Treatment modalities and recommendations. Prim Care 1983;10:443.

5. Dellon AL. Prediction of recurrence in incompletely excised basal cell carcinoma. Plast Reconstr Surg 1985;75:860.

6. Dubin N, Kopf AW. Multivariate risk score for recurrence of cutaneous basal cell carcinomas. Arch Dermatol 1983;119:373.

7. Jacobs GH, Rippey JJ, Altini M. Prediction of aggressive behavior in basal cell carcinoma. Cancer 1982;49:533.

8. Curry MC, Montgomery H, Winkelman RK. Giant basal cell carcinoma. Arch Dermatol 1977;113:316.

9. Leffel DJ, Headington JT, Wong DS, Swanson NA. Aggressivegrowth basal cell carcinoma in young adults. Arch Dermatol 1991;127:1663. 